\title{
The nonlinear diocotron mode in a pure electron plasma
}

\author{
Noel R. Corngold \\ California Institute of Technology, Pasadena, California 91125
}

(Received 12 March 1996; accepted 28 May 1996)

The nonlinear diocotron mode, discussed by Fine, Driscoll, and Malmberg [Phys. Rev. Lett. 63, 2232 (1989)] is characterized by two equations, one describing the frequency of orbiting, the other giving the quadrupole moment, as functions of size and offset. A new analysis, based on the method of moments, which yields equations more general in their content, is presented here. For example, the new equations describe columns whose shapes are not elliptical and whose densities are not constant. (C) 1996 American Institute of Physics. [S1070-664X(96)01409-7]

\section{INTRODUCTION}

This article is presented in the spirit of "if anything is worth doing it is worth doing to excess." The particular activity we are concerned with here is the analysis of a series of experiments on rotating columns of pure electron plasma which formed a large part of the 1988 doctoral research ${ }^{1}$ of Kevin Fine at the University of California, San Diego. The experimental results were published by Fine, Driscoll, and Malmberg (henceforth FDM) in Physics Review Letters. ${ }^{2}$ They were analyzed by Fine several years $\operatorname{later}^{3}$ in terms of a simple model based on an analogy with fluid dynamics. Then, Chu et al., in an article ${ }^{4}$ devoted to pure electron plasmas in asymmetric traps, showed that the principal results of Ref. 3 followed from the Hamiltonian dynamics of an elliptical plasma model which is a dynamical system with two degrees of freedom. Our point is that these elegant experiments present a clear challenge to "theory." The models used in Refs. 3 and 4 are quite simple (plasma columns with zero temperature, constant density, elliptical cross sections) yet surprisingly successful, though in the experiment the temperature is not zero, the density far from uniform, and the cross sections not precisely elliptical. It is not at all clear how these particular models used may be extended, to account for these effects. And it is certainly not clear why these simple models are so successful. In this article, we derive the principal results of Refs. 3 and 4 through approximations imposed upon a familiar and systematic treatment of the fluid equations for the electron plasma-through consideration of moments. The merits of our treatment are: (i) we produce useful expressions which are generalizations of those obtained in Refs. 3 and 4 and (ii) thinking about the approximations needed to reach this end gives us insight into the conditions under which the simple formulas are successful.

Let us be more specific. The simple theory produces two important results. The first is an equation relating the frequency at which the column rotates about the center axis of the cylinder, with the "offset" - the radius of the orbit traced by the center of the column-and the shape of the cross section of the column - the degree to which it has been deformed from a circular shape. We call this the "frequency equation."' It is

$$
\omega=\omega_{D}\left(1+d^{2}-\frac{1}{2} q_{2} r_{p}^{2}\right),
$$

with $\omega_{D}$ the "diocotron frequency," $\omega_{p}^{2} / 2 \omega, d=R / a$ a mea- sure of the offset versus the radius of the cylinder, $q_{2}$ a (normalized) quadrupole moment, a measure of the deformation of shape, and $r_{p}$ a measure of the cross-sectional area of the column (see Fig. 1). We defer more precise definition until later-in any case our notation will be slightly different. We shall find that this equation is quite general and comes easily, once we assume that offsets are modest.

The second equation, which we call the "quadrupole equation" relates the deformation to offset and to area (see Fig. 2). It is (deceptively) simple

$$
q_{2}=\frac{2 r_{p}^{2}}{\left(1-r_{p}^{2}\right)^{2}} d^{2}
$$

but turns out to be not so well founded as the first equation. Of course, the singularity at $r_{p}=1$ is spurious, the equation being valid only for small areas. And, the simple dependence upon offset is surprising. We shall find that reaching this equation requires heavier approximation.

Finally, before we begin the detailed calculations which produce the equations, we should repeat that they are obtained by Fine through transcription and reinterpretation of a result in the fluid mechanics of vortex patches, a result based on a picture of the column as elliptical in shape and having uniform electron density (vorticity). We will be able to relax those requirements somewhat, and to consider versions of the two equations which are generalized, somewhat. However, if one attempts to deal with a markedly different situation one has to abandon the notion that the experiments can be described by two, simple equations.

\section{ANALYSIS}

We contemplate a cold fluid of electrons not in the laboratory frame, but in a reference frame rotating with constant angular frequency $\Omega$ about the central, or $z$ axis of our system. We shall need the equation of continuity

$$
\frac{\partial n}{\partial t}+\boldsymbol{\nabla} \cdot(n \mathbf{u})=0, \quad n=n(\mathbf{r}, t), \quad \mathbf{u}=\mathbf{u}(\mathbf{r}, t),
$$




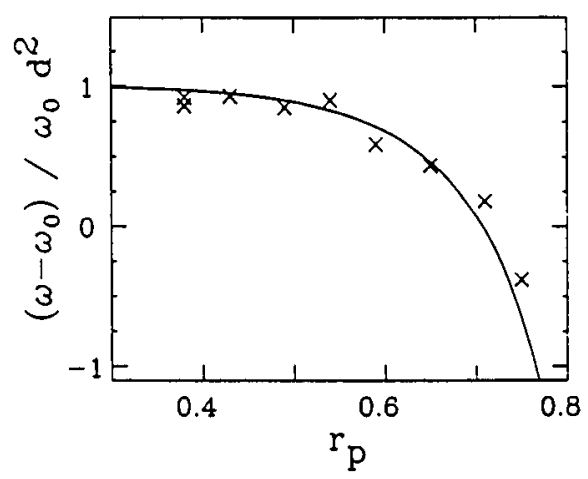

FIG. 1. This is Fig. 3 of Ref. 3, captioned "Measured fractional frequency shift over $d^{2}$ vs column radius...." The solid curve is the simple formula, our Eq. (1).

$$
\begin{aligned}
\frac{D \mathbf{u}}{D t} & +2 \boldsymbol{\Omega} \times \mathbf{u}+\boldsymbol{\Omega} \times(\boldsymbol{\Omega} \times \mathbf{r}) \\
& =\frac{q}{m}\left[\mathbf{E}(\mathbf{r}, t)+\frac{1}{c}[\mathbf{u} \times \mathbf{B}(\mathbf{r}, t)]=\mathscr{b}-(\boldsymbol{\omega} \times \mathbf{u}) .\right.
\end{aligned}
$$

We deal with a regime in which " $(v / c)^{2}$ terms", are neglected and with flows which lie in a plane perpendicular to the $z$ axis. The functions $\mathscr{C}$ (henceforth, "the field"), and $\boldsymbol{\omega}$, the cyclotron frequency, are taken to be independent of $z$. In our regime, the electric and magnetic fields in the rotating and laboratory frames are related by

$$
\mathbf{E}=\mathbf{E}_{0}+\frac{1}{c}\left[(\boldsymbol{\Omega} \times \mathbf{r}) \times \mathbf{B}_{0}\right], \quad \mathbf{B}=\mathbf{B}_{0}-\frac{1}{c}\left[(\boldsymbol{\Omega} \times \mathbf{r}) \times \mathbf{E}_{0}\right]
$$

(The subscript 0 denotes the laboratory frame; the components of $\mathbf{r}$ and $\mathbf{r}_{0}$ are related by the usual equations for rotating coordinates.) We consider experiments in which the magnetic field is uniform and parallel to the $z$ axis, and the fluid is confined by a circular, conducting cylinder, of radius $a$. In these circumstances, the momentum equation, Eq. (4), becomes

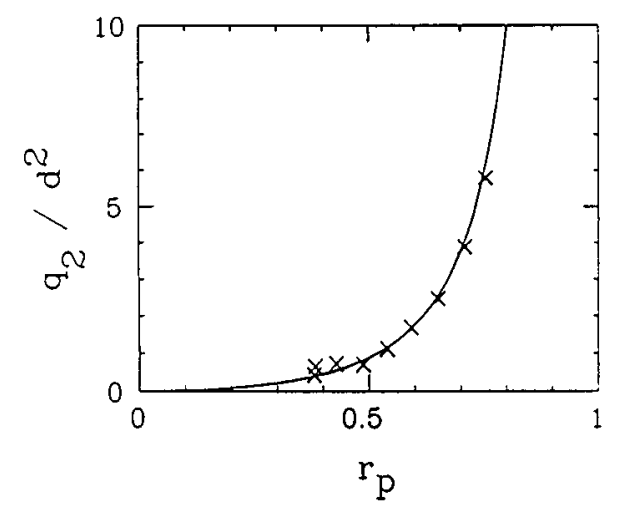

FIG. 2. This is Fig. 2 of Ref. 3, captioned "Measured quadrupole distortion over $d^{2}$ vs column radius... ." The solid curve is the simple formula, our Eq. (2).

$$
\frac{D \mathbf{u}}{D t}+(2 \boldsymbol{\Omega}+\boldsymbol{\omega}) \times \mathbf{u}=\mathscr{b}_{0}+\left(\Omega^{2}+\boldsymbol{\Omega} \cdot \boldsymbol{\omega}\right) \mathbf{r}
$$

where $\boldsymbol{\omega}$ now denotes the (constant) cyclotron frequency, $(q / m c) \mathbf{B}_{0}$. This is the equation we will study. We shall find that the frequency equation follows easily upon consideration of the motion of the center of mass of the column, while the quadrupole equation is obtained from moments of the equation of continuity.

\section{A. Center-of-mass motion}

If we recall that the position and velocity of the center of mass are defined by

$$
\int d \mathbf{r} n(\mathbf{r}, t) \mathbf{r}=N \mathbf{R}(t)
$$

and

$$
\frac{d}{d t} \int d \mathbf{r} n(\mathbf{r}, t) \mathbf{r}=N \mathbf{V}(t)
$$

we deduce from Eq. (6) that

$$
\begin{aligned}
\frac{d \mathbf{V}}{d t}+(2 \boldsymbol{\Omega}+\boldsymbol{\omega}) \times \mathbf{V}= & \frac{1}{N} \int d \mathbf{r} n(\mathbf{r}, t) \cdot \mathscr{b}_{0} \\
& +\left(\Omega^{2}+\boldsymbol{\Omega} \cdot \boldsymbol{\omega}\right) \mathbf{R} .
\end{aligned}
$$

We specialize further by limiting ourselves to columns which rotate rigidly and uniformly about the $z$ axis (no wobbling!) and whose internal flows are steady; the centers are at rest in our rotating frame. Setting $\mathbf{V}=0$ leads at once to the quite general "dispersion-equation",

$$
\left(\Omega^{2}+\boldsymbol{\Omega} \cdot \boldsymbol{\omega}\right)=-\frac{1}{N R^{2}} \int d \mathbf{r} n(\mathbf{r})\left[\mathbf{R} \cdot \mathscr{\ell}_{0}(\mathbf{r}, \mathbf{R})\right] .
$$

It is useful to regard the electric field as composed of two components, $\mathscr{C}_{k}=\mathscr{A}_{k}^{S}+\mathscr{C}_{k}^{W}$. The self field is due to the charges which constitute the column, while " $w$ "' denotes the contribution of the image or "wall" charges. Since the integral in Eq. (8) is proportional to the total force experienced by the column, the contribution of the self part of the electric field must integrate to zero in the equation; only the wall contribution remains. (Henceforth, we drop the zero subscript.)

An easy application of Eq. (8) now yields an important result. If we view our column as an assembly of line charges, the field due to the images of the assembly (wall) is known to be

$$
\mathscr{C}_{k}^{W}=\frac{1}{2} \omega_{p}^{2}\left(R_{k}+\frac{2}{a^{2}}\left(\hat{Q}+\hat{Q}_{R}\right)_{k l} r_{l}+\cdots\right) .
$$

In this equation the plasma frequency, $\omega_{p}^{2}$, is based upon the mean charge density present in the system, $\left(N q / \pi a^{2} L\right)$, not the mean density of charge in the column. We shall use the relation $N q^{2} / m L=\frac{1}{4} \omega_{p}^{2} a^{2}$ frequently. The matrix $\left(\hat{Q}_{R}\right)_{k l}=\left(R_{k} R_{l}-\frac{1}{2} \delta_{k l} R^{2}\right)$, while $\hat{Q}_{k l}$ is a traceless matrix of certain normalized quadrupole moments of the assembly, which we shall discuss later. (The first two terms of this 
multipole expansion make a good approximation if the assembly is neither too large nor too irregular.) Upon substituting Eq. (9) into Eq. (8) we obtain

$$
\Omega^{2}+\boldsymbol{\Omega} \cdot \boldsymbol{\omega}+\frac{1}{2} \omega_{p}^{2}\left(1+\frac{R^{2}}{a^{2}}+\frac{2}{a^{2} R^{2}}\left(\hat{Q} \hat{Q}_{R}\right)+\cdots\right)=0 .
$$

Equation (10) will become the frequency equation, the first of the two key equations used in the analysis of the experiments. It appears here under quite general circumstances, in no way connected with flat distributions, or elliptical shapes. It also has nothing to do with the nature of the flow. We may simplify by placing the center of mass-which is at restupon the $x$ axis, so that

$$
\left(\hat{Q}_{R}\right)_{k l}=Q_{R} \sigma_{k l}=\frac{R^{2}}{2} \sigma_{k l}, \quad \text { where } \sigma_{k l}=\left[\begin{array}{cc}
1 & 0 \\
0 & -1
\end{array}\right] .
$$

For many systems of simple shape, we will have $\hat{Q}_{k l}=Q \sigma_{k l}$. Then the frequency equation, Eq. (10) becomes

$$
\begin{aligned}
& \Omega^{2}+\boldsymbol{\Omega} \cdot \boldsymbol{\omega}+\frac{1}{2} \omega_{p}^{2}\left(1+\frac{2}{a^{2}}\left(Q+Q_{R}\right)+\cdots\right)=0, \\
& Q=\frac{1}{2} \int d^{2} \mathbf{x} \hat{n}(\mathbf{x})\left(x_{1}^{2}-x_{2}^{2}\right)
\end{aligned}
$$

"in the quadrupole approximation." If we assume that the cyclotron frequency is considerably larger than all other frequencies, and we recall the definition of the diocotron frequency $\omega_{D}=\frac{1}{2}\left(\omega_{p}^{2} / \omega\right)$, we see that Eq. (11) is identical with Eq. (1). (For details of notation, see ahead.)

\section{B. Moments of the density}

We turn, now, to the moments of the stationary distribution of charge. Since we are dealing, in fact, with a flow in two dimensions, it is convenient to switch from a threedimensional density of charge, $n(\mathbf{r})$ whose integral is $N$, to a two-dimensional density, $\hat{n}(\mathbf{x})$, whose integral over the cross section of the cylinder is unity. Further, we write $\mathbf{r}=\mathbf{R}+\mathbf{x}$, coordinates $x_{k}$ being referred to the center of mass. Consider, first, moments of the equation of continuity for a stationary flow, viewed in the rotating frame. We have

$$
\int d^{2} \mathbf{x}\left(1, x_{i}, x_{i} x_{j}, \ldots\right) \boldsymbol{\nabla} \cdot[\hat{n}(\mathbf{x}) \mathbf{u}(\mathbf{x})]=0 .
$$

The "zeroth" moment is trivially zero if the density falls to zero within the container, while the vanishing of the set of first moments reflects the fact that we measure relative to the center of mass. It is the set of second moments that interests us. The set yields a $(2 \times 2)$ matrix of equations

$$
\text { Sym } \mathscr{C l}_{i j} \equiv \frac{1}{2} \int d^{2} \mathbf{x} \hat{n}(\mathbf{x})\left(x_{i} u_{j}+x_{j} u_{i}\right)=0,
$$

with

$$
\mathscr{C}_{i j} \equiv \int d^{2} \mathbf{x} \hat{n}(\mathbf{x}) x_{i} u_{j}
$$

Their study is the central part of this article. The set will turn out to contain but a single, independent equation. That equation will be the second of the key equations used in the analysis of the experiments.

To obtain concrete results, we require a flow-field $\mathbf{u}(\mathbf{x})$. We shall use an approximate solution to Eq. (6) which is obtained, traditionally, via two slightly different routes.

(i) One declares, simply, that "inertial effects are negligible." This is the "geostrophic approximation" dear to meteorologists. The term $D \mathbf{u} / D t$, the acceleration of an element of mass, is set equal to zero. Or,

(ii) one examines the inertial term in some detail, writing

$$
\frac{D \mathbf{u}}{D t}=\frac{\partial \mathbf{u}}{\partial t}+\boldsymbol{\nabla}\left(\frac{1}{2} \mathbf{u}^{2}\right)+\widetilde{\boldsymbol{\omega}} \times \mathbf{u},
$$

to display explicitly the kinetic energy and the vorticity of the flow, $\widetilde{\boldsymbol{\omega}}=\boldsymbol{\nabla} \times \mathbf{u}$. Then, Eq. (6) becomes

$$
\frac{\partial \mathbf{u}}{\partial t}+(2 \boldsymbol{\Omega}+\boldsymbol{\omega}+\widetilde{\boldsymbol{\omega}}) \times \mathbf{u}=-\nabla\left[\Phi-\left(\frac{1}{2} \mathbf{u}^{2}+\frac{1}{2}\left(\Omega^{2}+\boldsymbol{\Omega} \cdot \boldsymbol{\omega}\right) \mathbf{r}^{2}\right)\right],
$$

with $\Phi$ the electrostatic-potential energy per unit mass. Since the flow is stationary, the geostrophic result follows upon the assumptions that the vorticity is small compared with the cyclotron frequency, $\boldsymbol{\omega}$, and that the kinetic energy is small compared with both the potential energy and the portion of the centrifugal potential that is proportional to $\boldsymbol{\omega}$. In this limit of large magnetic field, the equation for the flow becomes

$$
\boldsymbol{\omega} \times \mathbf{u}=-\nabla\left[\Phi-\frac{1}{2}(\boldsymbol{\Omega} \cdot \boldsymbol{\omega}) \mathbf{r}^{2}\right] .
$$

Thus, the flow in the rotating frame is " $E \times B$," corrected by $\boldsymbol{\Omega} \times \mathbf{r}$. (Note that it is easy to begin to take effects of temperature into account by adding an " $n k T$ " term to the quantities in square brackets.) In terms of components, we have

$$
\omega u_{j}=\epsilon_{j k}\left[\mathscr{C}_{k}+\omega \cdot \Omega r_{k}\right],
$$

where

$$
\epsilon_{j k}=\left[\begin{array}{cc}
0 & 1 \\
-1 & 0
\end{array}\right], \quad \tau_{j k}=\left[\begin{array}{ll}
0 & 1 \\
1 & 0
\end{array}\right] .
$$

We have introduced $\tau$, a third $2 \times 2$ matrix. The matrices $\sigma, \epsilon$, and $\tau$, along with the unit matrix, form a convenient basis for representing real $2 \times 2$ matrices. (Of course, they are related trivially to Pauli's matrices.) Note that $\sigma^{2}=\tau^{2}=-\epsilon^{2}=1$, $\sigma \tau=\epsilon, \sigma \epsilon=\tau$, and $\epsilon \tau=\sigma$. If we use Eq. (13), the moment equations, Eq. (12), become

$$
\begin{aligned}
& \operatorname{Sym} \mathscr{C}_{i j}=\operatorname{Sym} \epsilon_{j k} M_{i k}^{0}=0, \\
& M_{i k}^{0}=\int d^{2} \mathbf{x} \hat{n}(\mathbf{x}) x_{i}\left[\mathscr{b}_{k}+\omega \cdot \Omega r_{k}\right] .
\end{aligned}
$$

Our task is to extract from them, finally, the second of the key equations which fit the experiments so well. Here, we beg the reader's patience as we proceed through some rather dry manipulations. With "Sym" denoting the symmetric part of the matrix which lies to the right, we note that adding 
a multiple of the unit matrix to $M^{0}$ does not alter the equations. Thus, after writing $r_{k}=R_{k}+x_{k}$, we introduce the matrix $M_{i k}$, and obtain the important equation

$$
\text { Sym } \epsilon_{j k} M_{i k}=0, \quad M_{i k}=\int d^{2} \mathbf{x} \hat{n}(\mathbf{x}) x_{i} b_{k}+\omega \cdot \Omega \hat{Q}_{i k},
$$

with

$$
\hat{Q}_{i k}=\int d^{2} \mathbf{x} \hat{n}(\mathbf{x})\left[x_{i} x_{k}-\frac{1}{2} \delta_{i k} x^{2}\right] .
$$

As promised earlier, the traceless quadrupole matrix $\hat{Q}$ has been defined. For the systems we consider, $\hat{Q}_{i k}=Q \sigma_{i k}$, and $Q<0$.

[The traces of both the matrices, $\mathscr{C} 6$ and $M$, have simple, physical meaning. $\mathscr{L}_{k k}$, the trace of $\mathscr{L}$, is seen to be proportional to $-\hat{z} \cdot \int d^{2} \mathbf{x} \hat{n}(\mathbf{x})(\mathbf{x} \times \mathscr{\ell})$, itself proportional to the torque exerted by the electric field upon the column. The expression for "self-torque" may be seen to vanish, by symmetry, and the torque exerted by the image charges vanishes, too. $\mathscr{C} b$ is traceless. $M_{k k}$, the trace of $M$, does not vanish; it is proportional to the virial of the system of charges. That quantity is particularly simple for an assembly of line charges; the wall virial is also easy to compute, in the quadrupole approximation.]

The next step is mandated by the requirement that the analysis of experiments involves no moments higher than quadrupole. In view of Eq. (15), then, we must assume that linear variation of the electric field makes a good approximation, that we may neglect quadratic and higher terms. (As a consequence, Poisson's equation demands that the charge density not show great variation, either.) Thus, we are led to the approximation

$$
\mathscr{C}_{k}(\mathbf{x})=\mathscr{C}_{k}(0)+\omega_{p}^{2} \hat{U}_{k l} x_{l}+\cdots,
$$

which produces

$$
M_{i k}=\omega_{p}^{2} \hat{U}_{k l}\left(\hat{Q}_{i l}+\frac{1}{2} \delta_{i l}\left\langle x^{2}\right\rangle\right)+\omega \cdot \Omega \hat{Q}_{i k} .
$$

Note that we have encountered the approximation already, in the description of the wall contribution, Eq. (9). Generally, the matrix $\hat{U}$ is symmetric, and its trace is given by Poisson's equation. We shall see that it is diagonal, too. Thus, we may write

$$
M_{i k}=\omega_{p}^{2}\left(U_{0} \sigma_{k l}+U_{1} \delta_{k l}\right)\left(Q \sigma_{i l}+\frac{1}{2} \delta_{i l}\left\langle x^{2}\right\rangle\right)+\omega \cdot \Omega Q \sigma_{i k}
$$

and the evaluation of $\operatorname{Sym} \epsilon_{j k} M_{i k}$ leads to the simple scalar equation,

$$
\omega_{p}^{2}\left(\frac{1}{2}\left\langle x^{2}\right\rangle U_{0}+Q U_{1}\right)+\omega \cdot \Omega Q=0,
$$

or

$$
\left\langle x^{2}\right\rangle U_{0}+2 Q U_{1}-Q\left[1+\frac{2}{a^{2}}\left(Q+Q_{R}\right)\right]=0 .
$$

These equations are, in fact, the quadrupole equation albeit in abstract form. The discussion which follows fills in details.

We break $\hat{U}$ into two parts, $\hat{U}^{S}$ and $\hat{U}^{W}$; since

$$
\mathscr{C}_{k}^{W}=\frac{1}{2} \omega_{p}^{2}\left(R_{k}+\frac{2}{a^{2}}\left(\hat{Q}+\hat{Q}_{R}\right)_{k l} r_{l}+\cdots\right),
$$

we have $\left(\hat{U}^{W}\right)_{k l}=\left(1 / a^{2}\right)\left(\hat{Q}+\hat{Q}_{R}\right)_{k l}$.

For the column, the self-field is

$$
\begin{aligned}
\mathscr{C}_{k}^{S} & =2 \frac{N q^{2}}{m L} \int d^{2} \mathbf{y} \hat{n}(\mathbf{y})(\mathbf{x}-\mathbf{y})_{k} /(\mathbf{x}-\mathbf{y})^{2} \\
& =\frac{1}{2} \omega_{p}^{2} a^{2} \int d^{2} \mathbf{y} \hat{n}(\mathbf{y}) \frac{\partial}{\partial x_{k}} \log |\mathbf{x}-\mathbf{y}| .
\end{aligned}
$$

Then, we write

$$
\mathscr{C}_{k}^{S}(\mathbf{x})=\mathscr{C}_{k}^{S}(0)-\omega_{p}^{2} a^{2} \hat{T}_{k l} x_{l}+\cdots,
$$

where

$$
\begin{aligned}
\hat{T}_{k l} & =\int d^{2} \mathbf{x} \hat{n}(\mathbf{x}) \frac{1}{x^{4}}\left[x_{k} x_{l}-\frac{1}{2} \delta_{k l} x^{2}\right] \\
& =-\frac{1}{2} \int d^{2} \mathbf{x} \hat{n}(\mathbf{x}) \frac{\partial^{2}}{\partial x_{k} \partial x_{l}} \log |\mathbf{x}| .
\end{aligned}
$$

The $T$ matrix is not traceless $(\nabla \cdot \mathbf{E} \neq 0)$. It may be written as

$$
\begin{aligned}
& \hat{T}_{k l}=T \sigma_{k l}-\frac{\pi}{2} \hat{n}(0) \delta_{k l}, \\
& T=\frac{1}{2} \int d^{2} \mathbf{x} \hat{n}(\mathbf{x})\left(x_{1}^{2}-x_{2}^{2}\right) /\left(x_{1}^{2}+x_{2}^{2}\right)^{2} .
\end{aligned}
$$

With the introduction of $\hat{T}_{k l}$, Eq. (15) or Eq. (15d) becomes

$$
\begin{gathered}
a^{2}\left[\left\langle x^{2}\right\rangle T-\pi \hat{n}(0) Q\right]-\frac{\left\langle x^{2}\right\rangle}{a^{2}}\left(Q+Q_{R}\right) \\
+Q\left[1+\frac{2}{a^{2}}\left(Q+Q_{R}\right)\right]=0
\end{gathered}
$$

or

$y^{2}\left(Q+Q_{R}-a^{4} T\right)+Q \pi a^{2} \hat{n}(0)-Q\left[1+\frac{2}{a^{2}}\left(Q+Q_{R}\right)\right]=0$

with $y^{2}=\left\langle x^{2}\right\rangle / a^{2}$. If we compare these equations with Eq. $(15 \mathrm{c})$ we find

$$
U_{0}=\frac{1}{a^{2}}\left(Q+Q_{R}\right)-a^{2} T, \quad U_{1}=\frac{1}{2} \pi \hat{n}(0) a^{2} .
$$

\section{Closure}

Equation (20), while promising and true, is somewhat different from the successful quadrupole equation-it contains too many unknown quantities. We need to eliminate $\pi a^{2} \hat{n}(0)$ and $T$ - that is, express them in terms of $Q$ and $\left\langle x^{2}\right\rangle$. We begin with a simple argument, which is quite successful. Consider fairly flat distributions of near-elliptical shape, and cross-sectional area, $A$, then $\hat{n}(0) \approx 1 / A, A \approx 2 \pi\left\langle x^{2}\right\rangle$, lead to $2 \pi \hat{n}(0)\left\langle x^{2}\right\rangle \approx 1$. With the latter, Eq. (20) becomes

$$
y^{4}\left(Q-a^{4} T\right)-y^{2} Q\left[1+\frac{2}{a^{2}}\left(Q+Q_{R}\right)\right]+\frac{1}{2} Q=-y^{4} Q_{R} .
$$


Next, we argue that the expression for $T$ suggests a closure relation $\left\langle x^{2}\right\rangle^{2} T \approx \lambda Q$, where $\lambda$ is a pure number. In the case of an elliptical figure of uniform density-the choice of $F$ and of $C W$-where, incidentally, the quadrupole moment and the eccentricity are related through $Q \approx \frac{1}{4} \epsilon^{2}\left\langle x^{2}\right\rangle$, the closure with $\lambda=\frac{1}{4}$ is a very good approximation (see ahead.) Thereupon-the term $2\left(Q+Q_{R}\right)$ being considered small compared with $a^{2}$-Eq. (21) collapses into the simple-and surprisingly accurate-

$$
Q=-\frac{1}{\left[\frac{1}{2}-\left(\left\langle x^{2}\right\rangle / a^{2}\right)\right]^{2}}\left(\frac{\left\langle x^{2}\right\rangle}{a^{2}}\right)^{2} Q_{R},
$$

which will be recognized as the quadrupole equation, our second goal.

We shall now make the reasoning more precise, hoping to discover why the simple estimate appears to be so accurate. We evaluate $Q, T$, and $2 \pi \hat{n}(0)\left\langle x^{2}\right\rangle$ for a class of shapes and densities, to see how accurate the simple estimates are.

\section{Generalization}

We consider distributions more realistic than the "flat, elliptical" or "waterbag." Let

$$
n(\mathbf{x})=\int_{0}^{\infty} d t \mu(t) \delta[t-f(\mathbf{x})]=\mu[f(\mathbf{x})]
$$

be an un-normalized distribution whose level curves are those of the function $f(\mathbf{x})$. As an example we might choose

$$
f(\mathbf{x})=\frac{1}{a_{1}^{2}}\left(x_{1}^{2}+x_{2}^{2}\right)-\frac{\epsilon^{2}}{a_{1}^{2}} x_{2}^{2}=\xi\left(1-\epsilon^{2} \sin ^{2} \theta\right)
$$

producing level curves which are ellipses of fixed eccentricity, defined through $a_{2}^{2}-a_{1}^{2}=a_{2}^{2} \epsilon^{2}$. If we use conventional polar coordinates, with $\xi=\left(1 / a_{1}^{2}\right)\left(x_{1}^{2}+x_{2}^{2}\right)$, we obtain the second expression for $f(\mathbf{x})$. In fact, the analysis we shall present goes through easily for a more general class of level curves, namely,

$$
f(\mathbf{x})=B(\xi)\left[1-\epsilon^{2} p(\theta)\right], \quad p(\theta+2 \pi)=p(\theta) .
$$

(Of course, only those level curves which have the symmetries assumed earlier will be useful.) If we compute the quantities $\left\langle x^{2}\right\rangle, Q, T$, and $\hat{n}(0)$ for the level curves described by Eqs. (23) and (25), with-for example- $B(\xi)=\xi$, we find

$$
\begin{aligned}
\mathscr{N} \equiv \int d^{2} \mathbf{x} n(\mathbf{x}) & =\pi a_{1}^{2} \int_{0}^{\infty} d \xi \mu(\xi)\left\langle\frac{1}{1-\epsilon^{2} p(\theta)}\right\rangle \\
& =\pi a_{1}^{2} \lambda_{1}(\epsilon) \int_{0}^{\infty} d \xi \mu(\xi)
\end{aligned}
$$

and $\hat{n}(0)=\mu(0) / \mathscr{N}$.

$$
\begin{aligned}
\left\langle x^{2}\right\rangle & =\left(\pi a_{1}^{4} / \mathscr{N}\right) \int_{0}^{\infty} d \xi \xi \mu(\xi)\left\langle\frac{1}{\left[1-\epsilon^{2} p(\theta)\right]^{2}}\right\rangle \\
& =\left(\pi a_{1}^{4} / \mathscr{N}\right) \lambda_{2}(\epsilon) \int_{0}^{\infty} d \xi \xi \mu(\xi),
\end{aligned}
$$

$$
\begin{aligned}
Q & =\left(\pi a_{1}^{4} / 2 \mathscr{N}\right) \int_{0}^{\infty} d \xi \xi \mu(\xi)\left\langle\frac{\cos 2 \theta}{\left[1-\epsilon^{2} p(\theta)\right]^{2}}\right\rangle \\
& =-\left(\pi a_{1}^{4} / 4 / N\right) \epsilon^{2} \lambda_{3}(\epsilon) \int_{0}^{\infty} d \xi \xi \mu(\xi), \\
T & =-(\pi / 2 /) \mu(0)\left\langle\cos 2 \theta \log \left[1-\epsilon^{2} p(\theta)\right]\right\rangle \\
& =-(\pi / 8 / N) \epsilon^{2} \lambda_{4}(\epsilon) \mu(0) .
\end{aligned}
$$

The angular brackets denote angle averages. We have a nice factoring into two components, one connected with the shape of the level curves, the other with the density profile. We infer, easily,

$$
Q=-\frac{1}{4} \epsilon^{2} \frac{\lambda_{3}}{\lambda_{2}}(\epsilon)\left\langle x^{2}\right\rangle
$$

and

$$
\frac{\lambda_{3}}{\lambda_{2} \lambda_{4}}(\epsilon)\left\langle x^{2}\right\rangle^{2} T=2 \pi \hat{n}(0)\left\langle x^{2}\right\rangle \frac{1}{4} Q,
$$

the second of these being quite suggestive, in the light of the simple arguments made earlier.

Also,

$$
2 \pi \hat{n}(0)\left\langle x^{2}\right\rangle=2 \frac{\lambda_{2}(\epsilon)}{\lambda_{1}^{2}(\epsilon)} \frac{\mu(0) \mu_{1}}{\mu_{0}^{2}},
$$

with

$$
\mu_{k}=\int_{0}^{\infty} d \xi \xi^{k} \mu(\xi)
$$

Note that as $\epsilon \rightarrow 0$ all quantities $\lambda_{k}$ are $O(1)$. The terms $\lambda_{1}(0)$ and $\lambda_{2}(0)$ are precisely unity, while $\lambda_{3}(0)$ and $\lambda_{4}(0)$ are unity in the particular case of elliptical level curves. In that case

$$
\begin{aligned}
& \lambda_{1}(\epsilon)=\frac{1}{\sqrt{1-\epsilon^{2}}}, \quad \lambda_{2}(\epsilon)=\frac{1-\frac{1}{2} \epsilon^{2}}{\left(1-\epsilon^{2}\right)^{3 / 2}}, \\
& \lambda_{3}(\epsilon)=\frac{1}{\left(1-\epsilon^{2}\right)^{3 / 2}}, \quad \lambda_{4}(\epsilon)=\frac{4}{\left[1+\sqrt{ } 1-\epsilon^{2}\right]^{2}} . \\
& \frac{\lambda_{3}}{\lambda_{2} \lambda_{4}}(\epsilon)=\frac{1}{2} \frac{\left[1+\sqrt{1-\epsilon^{2}}\right]^{2}}{1-\frac{1}{2} \epsilon^{2}}=1+O\left(\epsilon^{4}\right), \\
& \frac{\lambda_{2}}{\lambda_{1}^{2}}(\epsilon)=\frac{1-\frac{1}{2} \epsilon^{2}}{\left(1-\epsilon^{2}\right)^{1 / 2}}=1+O\left(\epsilon^{4}\right) .
\end{aligned}
$$

What do these results say? The first of Eq. (30) simply connects quadrupole moment, second moment, and eccentricity for the shapes we consider. The second equation provides one of the two relations of closure needed to reduce Eq. (20) to Eq. (22). The key quantity, $\left(\lambda_{3} / \lambda_{2} \lambda_{4}\right)(\epsilon)$, is seen to deviate from unity by terms $O\left(\epsilon^{4}\right)$-in the case of elliptical shapes. Setting it equal to unity, as we did in the previous discussion, appears to be a very good approximation. The second closure relation, Eq. (31), displays a factor, $\left(\lambda_{2} / \lambda_{1}^{2}\right)$, which also differs from unity by $O\left(\epsilon^{4}\right)$ - a remarkable result. We begin to see why the simple quadrupole formula works so well. 
Let us exploit the weak dependence upon eccentricity by ignoring it. Then, the general closure relations may be written

$$
2 \pi \hat{n}(0)\left\langle x^{2}\right\rangle=\Theta, \quad\left\langle x^{2}\right\rangle^{2} T=\Theta \frac{1}{4} Q
$$

with

$$
\Theta \equiv 2 \frac{\mu(0) \mu_{1}}{\mu_{0}^{2}} .
$$

The crucial quantity, $\Theta$, depends upon the density profile. Now, reduction of the key equation, Eq. (20), yields

$$
Q\left(\left(y^{2}-\frac{1}{2}\right)^{2}+\frac{1}{4}(\Theta-1)-\frac{2}{a^{2}}\left(Q+Q_{R}\right) y^{2}\right)=-y^{4} Q_{R}
$$

which should be compared with Eq. (28). Calculation shows that if the density profile is

$$
\begin{aligned}
& \text { Flat("Water bag") then } \Theta=1 \text {, } \\
& \text { Gaussian in } \xi \quad \Theta=4 / \pi, \\
& \text { Exponential in } \xi \quad \Theta=2 .
\end{aligned}
$$

A remarkable aspect of the ratio, $\Theta$, is its "independence of scale;" should the density assume the form $\mu(\xi / \sigma)$, the ratio is seen to be independent of $\sigma$.

Before turning to Fine's experiments, we wish to discuss another aspect of our analysis, the behavior of our equations as $R \rightarrow 0$. Then the column is centered on the axis of the cylinder, and we expect the motion with frequency $\Omega$ to become one of the low-frequency modes familiar from the linear analysis of the dynamics of the column. ${ }^{5}$ Thus, if we consider the case of constant density we expect to connect with the equation

$$
\Omega_{*}(m)=\omega_{D}^{*}\left[m-1+\left(\frac{b}{a}\right)^{2 m}\right]
$$

describing the temporal variation of fields. Here the diocotron frequency is based on the density in the column, not - as we have chosen above - the mean density in the cylinder.

Consider two, different, limiting procedures. In the first we conclude from Eq. (22) that $Q$ vanishes as $Q_{R}$. Using that observation in the frequency equation, Eq. (11), leads us-with the neglect of terms $O\left(\Omega^{2}\right)$ - to the limiting behavior

$$
\Omega \rightarrow \omega_{D}=\omega_{D}^{*}\left(\frac{b}{a}\right)^{2}=\Omega_{*}(1) .
$$

It would appear that our equations describe a nonlinear extension of the $m=1$ mode. On the other hand, suppose we ignore Eq. (22) and, in Eq. (20), set $Q_{R}=0$ and re-introduce $(\omega \cdot \Omega)$. Equation (20) becomes

$$
\frac{1}{2} \omega_{p}^{2}\left(\frac{\left\langle x^{2}\right\rangle}{a^{2}}\left(Q-a^{4} T\right)+\pi \hat{n}(0) a^{2} Q\right)+(\omega \cdot \Omega) Q=0
$$

and if we "close," in the spirit of Eq. (33), we have

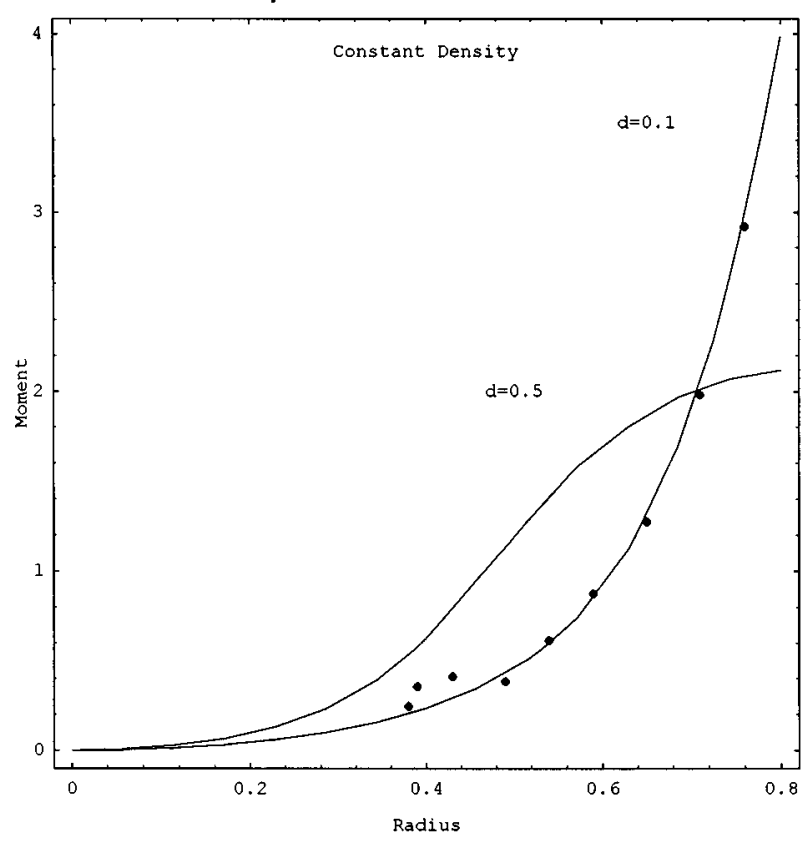

FIG. 3. Graph of the "full" quadrupole equation, Eq. (34), for constant density and elliptical boundary. "Radius" is $r_{p}$ while "moment" is $q_{2} / 2 d^{2}$.

$$
\frac{1}{2} \omega_{p}^{2}\left(\left[\frac{\left\langle x^{2}\right\rangle}{a^{2}}\right]^{2}+\frac{1}{4} \Theta\right)+(\omega \cdot \Omega)=0 .
$$

In the special case of uniform density, and a column of radius $b,\left\langle x^{2}\right\rangle=\frac{1}{2} b^{2}, \Theta=1$, and

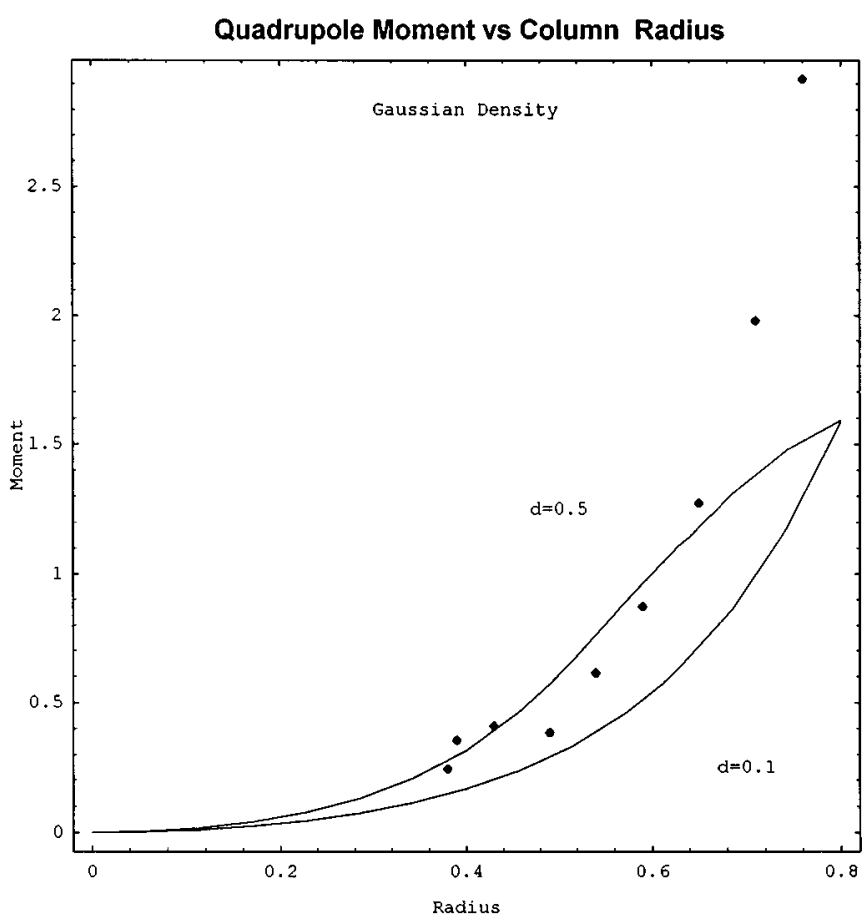

FIG. 4. Graph of the full quadrupole equation, Eq. (34), for Gaussian density and elliptical level curves. Radius is $r_{p}$ while moment is $q_{2} / 2 d^{2}$. 


$$
|\Omega| \rightarrow \frac{1}{2} \omega_{D}^{*}\left[1+\left(\frac{b}{a}\right)^{4}\right]=\frac{1}{2} \Omega *(2)
$$

the frequency of rotation becoming half the linear frequency of the signal received by a detector. This is as it should be, for a rotating column whose surface is deformed as $\cos m \theta$ will, when rotating at frequency $f$, signal at a frequency $m f$. Thus, our moment equations also describe, approximately, the nonlinear extension of the linear mode $m=2$.

But our equations describe nonuniform densities too, and one might ask whether Eq. (36) is useful in predicting the frequencies of stable, $m=2$ modes in general. Here, one runs into the issue of "quasi-modes," ",6 for all modes possess a damping caused by a species of "phase mixing." Perhaps, then, Eq. (36) will yield the real part of the complex mode. It turns out that while the limiting behavior of Eq. (36) is correct, its numerical accuracy is unimpressive.

Finally, I note another limit possessed by these equations. Let the plasma column have uniform density and elliptical cross-section and the containing cylinder have very large radius. Let the column rotate above its central axis, which coincides with the central axis of the cylinder. Then Eq. (35) and Eq. (32) give

$$
\Omega=-\omega_{D}^{*} \frac{2 a_{1} a_{2}}{\left(a_{1}+a_{2}\right)^{2}}
$$

which is a simple transcription of a result in fluid dynamics obtained by Kirchhoff, ${ }^{7}$ more than a century ago.

\section{EXPERIMENTS}

About notation. FDM describe offset by $d^{2}=R^{2} / a^{2}$ and the size of the column by $R_{p}$, the distance from the center at which the density has fallen to one half of its central value; $R_{p}^{2} / a^{2}=r_{p}^{2}$. We describe the size by $\left\langle x^{2}\right\rangle$. Thus the relation between $R_{p}^{2}$ and $\left\langle x^{2}\right\rangle$ depends upon the distribution of electron density. For example, given an ellipse of constant density, taking $R_{p}^{2}$ as the averaged (square) distance from center to edge gives $\left\langle x^{2}\right\rangle=\frac{1}{2} R_{p}^{2}$; if the density were Gaussian, we would have the slightly different $\left\langle x^{2}\right\rangle=0.48 R_{p}^{2}$. We shall use the former relation throughout. The quadrupole moments are related by $-2 Q / a^{2}=\frac{1}{2} r_{p}^{2} q_{2}, q_{2}$ being the quadrupole moment scaled, roughly, by the cross-sectional area of the column. Finally, Ref. 1 describes the deformation of cross section in terms of eccentricity $\epsilon$, where $\epsilon^{2}=-2 Q /\left\langle x_{2}\right\rangle^{2}$. One can show that $\epsilon^{2}=2 q_{2} \phi$, with $\phi$ independent of density profile $(\mu)$ for densities of the sort described above. In fact, $\phi=1+O\left(\epsilon^{2}\right)$ and may be set equal to unity unless the deformations are very large.
What can we contribute to the interpretation of the data? The simple theory, displayed in Fig. 2, does well enough; the more precise Eq. (34) contains the term $\left(2 / a^{2}\right)\left(Q+Q_{R}\right) y^{2}$ which is neglected in the derivation of the simple equation, Eq. (22), and the parameter $\Theta$, which characterizes the density distribution. Inclusion of these produces two notable consequences. First, the spurious divergence at $y^{2}=\frac{1}{2}$ is removed. Second, the equation has the special solution $Q=-Q_{R}=-\frac{1}{2} R^{2}$ for columns having $y^{2} \equiv\left\langle x^{2}\right\rangle / a^{2}=\Theta / 4$. As a consequence, the reduced quadrupole moment $q_{2} / d^{2}$ is not the universal function of $r_{p}$, independent of offset as Eq. (22) suggests. Rather, one finds a family of functions (graphs) labeled by offset (see Figs. 3 and 4). Further, the special solution constrains all graphs in the family to have the common point $r_{p}^{2}=\Theta / 2, q_{2} / d^{2}=4 / \Theta$, where they cross. When, instead, one plots $q_{2}$ vs $d$ for various fixed column sizes, $r_{p}$, using eccentricity rather than $q_{2}$, the special solution predicts that one curve, labeled $r_{p}=\sqrt{ } \mathrm{\Theta} / 2$, will be a straight line of slope $\sqrt{ } 8 / \Theta$, while those graphs labeled by sizes smaller, or larger than the critical value will be curved. Something like that appears in Fig. 4.3 of Ref. 1, but the agreement is "suggestive" rather than quantitative. Though the effect is small, it would-in principle-enable one to infer $\Theta$, and something about the density profile. Finally, a more important question: how does $\Theta$ affect our results? Though $\Theta$ has the peculiar property of being independent of scale, intrinsically "flatter" profiles yield smaller $\Theta$, and larger $q_{2}$. In fact, as Figs. 3 and 4 indicate, $\Theta=1$, which corresponds to a constant density (water bag), gives the best fit with experiment, though it hardly corresponds to reality. It is merely an indication that other, significant effects remain to be included in the analysis.

\section{ACKNOWLEDGMENTS}

Valuable conversations with my colleague Roy Gould have added much to this work. And I am grateful to Kevin Fine for his permission to use illustrations from his article, Ref. 3.

${ }^{1}$ K. S. Fine, Ph.D. dissertation, University of California, San Diego, 1988. ${ }^{2}$ K. S. Fine, C. F. Driscoll, and J. H. Malmberg, Phys. Rev. Lett. 63, 2232 (1989).

${ }^{3}$ K. S. Fine, Phys. Fluids B 4, 3981 (1992).

${ }^{4}$ R. Chu, J. S. Wurtele, J. Notte, A. J. Peurrung, and J. Fajans, Phys. Fluids B 5, 2378 (1993).

${ }^{5}$ R. J. Briggs, J. D. Daugherty, and R. H. Levy, Phys. Fluids 13, 421 (1970). ${ }^{6}$ N. Corngold, Phys. Plasmas 2, 620 (1995).

${ }^{7}$ H. Lamb, Hydrodynamics (Cambridge University Press, Cambridge, 1932). 